\title{
Patient-Controlled Sedation and Analgesia with Propofol and Alfentanil: A Preliminary Safety Evaluation Prior to Use of Non-Anaesthesiology Doctors
}

\author{
Andreas Nilsson $^{1 *}$, Folke Sjöberg ${ }^{1}$, Susanne Öster ${ }^{1}$, Hanne Bek-Jensen ${ }^{2}$, Claes Lennmarken ${ }^{1}$ \\ ${ }^{1}$ Department of Anesthesiology and Intensive Care, Linköping University Hospital, Linköping, Sweden; ${ }^{2}$ Department of Urology, \\ Linköping University Hospital, Linköping, Sweden. \\ Email: *andreas.nilsson@lio.se
}

Received November $23^{\text {rd }}, 2011$; revised December $9^{\text {th }}, 2011$; accepted December $18^{\text {th }}, 2011$

\begin{abstract}
Background: The aim was to evaluate safety aspects of patient-controlled sedation and analgesia (PCS) for extracorporeal shockwave lithotripsy (ESWL) and PCS to be handled by non-anaesthesiology doctors. Methods: Thirty-four ASA I-III patients used PCS with propofol and alfentanil for ESWL in this interventional study. Strict safety limits were defined regarding respiratory rate (RR), heart rate (HR), mean arterial blood pressure (MAP), oxygen saturation from pulse oximetry $\left(\mathrm{SpO}_{2}\right)$, and transcutaneous partial pressures of oxygen $\left(\mathrm{PtcO}_{2}\right)$ and carbon dioxide $\left(\mathrm{PtcCO}_{2}\right)$. The patients' levels of consciousness was graded on a five-point scale and monitored with Bispectral Index (BIS). A nurse anaesthetist was supervising the procedure but was instructed to intervene only if safety limits were breached. No supplementary oxygen was given. Results: All patients responded to verbal stimuli during treatment. Cardiovascular stability was maintained, but respiratory variables were affected. Two patients with $\mathrm{SpO}_{2}<90 \%$ and two cases of RR $\leq 8$ were diagnosed, and seven patients became hypercarbic $\left(\mathrm{PtcCO}_{2} \geq 6.5 \mathrm{kPa}\right)$. In 18 patients hypoxaemia was indicated as $\mathrm{PtcO}_{2} \leq 8.0 \mathrm{kPa}$. All these 18 patients were given supplementary oxygen. There was no correlation between dose of drugs, age, weight or any vital variable. The 34 patients would use PCS again in the case of future treatment. Conclusions: During ESWL treatment PCS can be used with good patients' satisfaction, and maintained cardiovascular stability, but PCS had an indisputable effect on pulmonary function with hypoxemia (resulting in need for supplementary oxygen) or hypercarbia. The person in charge of PCS must therefore be trained to perform according to the guidelines for sedation and/or analgesia by non-anaesthesiology doctors.
\end{abstract}

Keywords: Anaesthesia; Patient-Controlled Sedation; Safety; European Guidelines

\section{Introduction}

Extracorporeal shock wave lithotripsy (ESWL) is a noninvasive treatment for urethral and renal stones that uses acoustic shock waves to break stones into small fragments that can be excreted. Analgesia or sedation, or both, is required, as the procedure causes pain at the entry site of the shock waves and deep visceral discomfort $[1,2]$. The ESWL treatment is in most cases an outpatient treatment with a duration of less than one hour $[3,4]$. Midazolam/fentanyl is widely used which sometimes results in a long recovery period for patients. An alternative safe analgesia and sedation regime that offers more rapid recovery is therefore desirable. Patient-controlled sedation (PCS) with propofol and alfentanil has a fast onset time, and a short acting time, and it is possible for patients to titrate doses, to meet their judgment and need

\footnotetext{
${ }^{*}$ Corresponding author.
}

for treatment of pain and discomfort. PCS has been used for colonoscopy [5], cataract surgery [6], and transvaginal oocyte retrieval [7]. When PCS has been evaluated in terms of depth of sedation, cardiopulmonary signs, analgesia, recovery times and comfort for patients, the PCS method has been proved to be safe, $[1,8]$ and suitable for examination or treatment [8-10].

The aim of the present pilot study was to evaluate whether PCS can be implemented as a sedation routine for ESWL in accordance with the European Union of Specialists guidelines for sedation or analgesia by nonanaesthesiology doctors [11].

\section{Materials and Methods}

Thirty-four ASA class I-III patients between 18 and 90 years of age with renal stones were enrolled for elective extracorporeal shockwave lithotripsy (ESWL). Informed 
consent was obtained from each patient and pursuant to the recommendation of the local ethics committee. Patients were excluded if they were unable to understand the PCS-pump device and how to use it, if they misused drugs or if they were allergic to any of the study medications.

The ESWL treatment was done using a Lithotripter S (Dornier Med Tech, Kennesaw, USA). Before the procedure patients were given information about the procedure and the use of the patient-controlled pump device (Graseby 9300 PCS, Graseby Medical Ltd, Watford, UK). The patients were instructed to push the delivery button when they felt pain or discomfort. Pain was assessed using a visual analogue scale (VAS), on which 0 indicated no pain and 10 unbearable pain. After being positioned for ESWL the monitoring devices were applied to each patient. Data were collected for non-invasive mean arterial blood pressure (MAP), oxygen saturation $\left(\mathrm{SpO}_{2}\right)$, transcutaneously measured partial pressure of oxygen $\left(\mathrm{PtcO}_{2}\right)$ and carbon dioxide $\left(\mathrm{PtcCO}_{2}\right)$, sedation state, and respiratory rate (RR) with a fibre-optical monitor (Optovent ${ }^{\mathrm{TM}} \mathrm{RR}$ 9700, Optovent AB, Bromma, Sweden). Fibre-optical technique used for respiratory rating has sufficient accuracy for clinical monitoring [12].

To monitor depth of sedation, two methods were evaluated to ensure patients' safety during the trial. 1) Bispectral Index Monitoring (BIS) using A-2000 BIS ${ }^{\mathrm{TM}}$ system (Aspect Medical Systems, Natick, MA, USA) which has been proved as a method for estimating depth of sedation $[13,14]$; and 2) manual estimates of depth of sedation taken by the nurse anaesthetist using a modified five-levelled sedation scale used in a earlier study [15] and (Table 1). Both methods are well known to the staff and used daily.

$\mathrm{PtcO}_{2}$ and $\mathrm{PtcCO}_{2}$ were collected with TCM3-TINA (Radiometer, Copenhagen, Denmark) that has been used previously [16-19]. All values were monitored continuously and recorded at five-minute intervals, or in case of a rapid change.

A nurse anaesthetist was constantly supervising and present during the entire procedure. In addition, an anaesthesiologist was present on call but was never required during the study. Patients fasted for at least 6 hours.

The drugs used, comprised a solution of an analgesic (alfentanil $75 \mu \mathrm{g} / \mathrm{ml}$ ) and a sedative (propofol $8.5 \mathrm{mg} / \mathrm{ml}$ ) as had been used in a previous study on PCS for colonoscopy [20]. A bolus was given when the patient pressed the delivery button. If the demand was successful, a dose of $0.5 \mathrm{ml}$ of solution was infused over a period of $15 \mathrm{sec}-$ onds. Each dose contained alfentanil $0.0375 \mathrm{mg}$ and propofol $4.25 \mathrm{mg}$. No lockout period was used but demands made during active infusion were ignored. All demands, delivered or undelivered, were recorded. Before starting the ESWL procedure, all patients were given a loading dose of propofol $(0.3 \mathrm{mg} / \mathrm{kg})$ and alfentanil (2 $\mu \mathrm{g} / \mathrm{kg}$ ).

All safety limits were set before the procedure (Table 2). The patients were breathing air. If the $\mathrm{SpO}_{2}$ dropped below $90 \%$, or respiratory frequency fell below 8 /minute, supplementary oxygen, $3 \mathrm{~L} / \mathrm{min}$, was given through a nose catheter.

At the end of the procedure the patients were observed according to the routines in the post anaesthesia care unit: that is until they were fully alert, vital signs were stable, pain control was adequate, urination was normal and they were able to walk unaided.

Data are presented as mean (SD). Coefficients of correlation were made, to assess correlations between characteristics of patients, surveillance, and doses of drugs. Values of $r \geq 0.5$ were accepted as if there was a correlation. Probabilities of less than 0.05 were accepted as significant. For the statistical analysis we used Statistica ${ }^{\circledR}$ version 6.1 (Stat Soft Inc, Tulsa, USA).

\section{Results}

Thirty-four patients filled the qualification criteria and were enrolled for ESWL treatment during the study period, no patients were excluded. Patients' characteristics and measurements are shown in Tables 3 and 4 . The mean duration of the procedure was $31 \pm 6$ minutes. It was possible to treat all patients with ESWL and there

Table 1. Sedation scale.

\begin{tabular}{cl}
\hline Score & \multicolumn{1}{c}{ Degree of sedation } \\
\hline 1 & Fully awake and oriented \\
2 & Drowsy \\
3 & Eyes closed, responds promptly to verbal commands \\
4 & Eyes closed, rousable on mild physical stimulation only \\
5 & Eyes closed, unrousable on mild physical stimulation \\
\hline
\end{tabular}

Sedation scale modified from [16].

Table 2. Safety limits set before the procedure.

\begin{tabular}{cc}
\hline Mean arterial pressure & $\geq 70 \mathrm{mmHg}$ \\
Heart rate & $\geq 50$ \\
Respiratory rate & $\geq 8$ \\
Oxygen saturation & $\geq 90 \%$ \\
$\mathrm{PtcCO}_{2}$ & $\leq 6.5 \mathrm{kPa}$ \\
$\mathrm{PtcO}_{2}$ & $\geq 8.0 \mathrm{kPa}$ \\
\hline
\end{tabular}

Respiratory rate $=$ breaths/minute. Heart rate $=$ beats $/$ minute; $\mathrm{PtcCO}_{2}=$ Transcutaneous pressures of carbon dioxide; $\mathrm{PtcO}_{2}=$ Transcutaneous pressures of oxygen. 
Table 3. Details of patients. Data are expressed as mean (SD) or $n$.

\begin{tabular}{cc}
\hline Age $(\mathrm{yr})$ & $55(15.3)$ \\
Male/Female ratio & $21 / 13$ \\
Weight $(\mathrm{kg})$ & $76(11.5)$ \\
\hline
\end{tabular}

Table 4. Operative measurements. Data are expressed as mean (SD) or $n$.

\begin{tabular}{lcc}
\hline & Mean & Range \\
\hline Mean arterial pressure (mmHg) & $105(15.5)$ & $141 / 65$ \\
Heart rate & $65(11.3)$ & $102 / 40$ \\
Respiratory rate & $14.4(3.5)$ & $23 / 4$ \\
Oxygen saturation & $95(2.0)$ & $97 / 85$ \\
$\mathrm{PtcCO}_{2}$ & $5.3(1.4)$ & $9.7 / 1.9$ \\
$\mathrm{PtcO}_{2}$ & $8.8(1.9)$ & $15.5 / 5.0$ \\
Sedation score & $1.6(1.8)$ & $3 / 1$ \\
Bispectral index & $86.5(8.6)$ & $98 / 68$ \\
VAS & $4.2(1.6)$ & $8 / 1$ \\
Induction dose of Propofol (mg) & $22.1(3.4)$ & $30 / 17$ \\
Induction dose of Alfentanil (mg) & $0.13(0.02)$ & $0.20 / 0.11$ \\
Total dose of Propofol (mg) & $66.3(35.7)$ & $145 / 9$ \\
Total dose of Alfentanil (mg) & $0.60(0.32)$ & $1.28 / 0.08$ \\
Demands for PCS & $27.7(26.5)$ & $123 / 3$ \\
Deliveries of PCS & $15.5(8.4)$ & $34 / 2$ \\
\hline
\end{tabular}

Respiratory rate $=$ breaths $/$ minute. Heart rate $=$ beats $/$ minute; $\mathrm{PtcCO}_{2}=$ Transcutaneous pressures of carbon dioxide $(\mathrm{kPa}) ; \mathrm{PtcO}_{2}=$ Transcutaneous pressures of oxygen $(\mathrm{kPa})$.

were no complaints about PCS. All patients would have chosen PCS if they required a future lithotripsy.

Correlations were sought between patients' characteristics, surveillance data, and doses of drugs, without any results stronger than $r=0.5$, except for BIS data and sedation score.

\subsection{Cardiopulmonary Data}

Mean arterial blood pressure (MAP) decreased during the procedure; in one patient the recording was below the safety limit of 70 . During the procedures, 8 episodes of low heart rate were recorded in 3 patients, none which was below 40 .

Mean respiratory rate fell during the procedure and recordings below 10 breaths/min were found in $10 \mathrm{pa}$ tients demanding supplementary oxygen. Of these, 2 patients had a respiratory rate of 8 or less. The lowest respiratory rate recorded was 4 breaths $/ \mathrm{min}$. Two patients had 4 episodes of $\mathrm{SpO}_{2}$ of less than $90 \%$.
Mean transcutaneous partial pressure of oxygen in blood fell and carbon dioxide concentration rose during the procedure. With an upper safety limit of $\mathrm{PtcCO}_{2} \leq 6.5$ $\mathrm{kPa}, 7$ patients were recorded as having hypercarbia. With a lower safety limit of $\mathrm{PtcO}_{2} \geq 8 \mathrm{kPa}$, we recorded 64 episodes of hypoxia in 18 patients. All patients who were given supplementary oxygen had their saturation and $\mathrm{PtcO}_{2}$ returned to normal limits. In total, eighteen of the 34 patients showed signs of impaired ventilation as indicated by oxygen desaturation, tissue hypoxaemia, hypercarbia, or low respiratory rate.

\subsection{Covariance}

Five patients were outside the safety limits of more than one variable (Table 5).

Four of the 5 patients had a maintained stable circulation. The fifth patient had an initially low MAP (65 $\mathrm{mmHg}$ ), which increased during the procedure. The remaining patients were outside the limit for just one variable.

\subsection{Sedation and Pain}

Seven episodes of a BIS score between 68 and 70 were recorded in 7 patients. In 15 patients, there were 37 episodes of a manually estimated depth of sedation of 3 . No patient had a score of 4 . The correlation between BIS and sedation scores was $r=0.6$. The mean VAS score was 4.2 with one outlier at 8 .

\subsection{Data from the Pump Device}

The mean ratio between demanded and effectual doses was 1.77; in other words, the patients had a deficit in demanded doses corresponding to $77 \%$. Three patients had a ratio of more than 3.8 .

\section{Discussion}

We found that using PCS and a combination of propofol and alfentanil leads to that more than $50 \%$, of patients

Table 5. Measurements from the five patients who went over two safety limits.

\begin{tabular}{ccccc}
\hline Case no & Variable & Value & Variable & Value \\
\hline 1 & $\mathrm{PtcO}_{2}$ & 6.9 & $\mathrm{PtcCO}_{2}$ & 7.9 \\
2 & $\mathrm{PtcO}_{2}$ & 7.0 & $\mathrm{PtcCO}_{2}$ & 7.0 \\
3 & $\mathrm{PtcO}_{2}$ & 5.4 & Respiratory rate & 7 \\
4 & $\mathrm{PtcO}_{2}$ & 6.0 & Respiratory rate & 7 \\
5 & Oxygen saturation & 85 & $\mathrm{PtcO}_{2}$ & 6.5 \\
\hline
\end{tabular}

Respiratory rate $=$ breaths/minute; $\mathrm{PtcCO}_{2}=$ Transcutaneous pressures of carbon dioxide $(\mathrm{kPa}) ; \mathrm{PtcO}_{2}=$ Transcutaneous pressures of oxygen $(\mathrm{kPa})$. 
breathing air had insufficient respiratory function monitored as oxygen saturation $<90 \%$ or $\mathrm{PtcO}_{2}<8.0 \mathrm{kPa}$ ) or hypercarbia $\left(\mathrm{PtcCO}_{2}>6.5 \mathrm{kPa}\right)$. Cardiovascular function (MAP) was not affected. The negative respiratory effects of the sedation technique were easily corrected by uncomplicated action of non-anaesthesiology personal adding oxygen and communicating with the patient. Given this outcome, the present study supports this type of technique and protocol for sedation by non-anaesthesiology doctors and nurses. This technique (with added oxygen) performed by non-anaesthesiology doctors was implemented at our institution after the completion of the study and no complications or adverse events related to PCS has then been registered.

The present investigation is based on the assumption that it is difficult to stage a large multicenter trial assessing the safety of PCS for e.g., ESWL by examining adequate endpoints, such as significant anaesthetic complications. Instead an approach was selected in which we chose to thoroughly examine potential circulatory and respiratory effects by meticulously assessing both cardiovascular (repeated blood pressure measurements) and respiratory effects (saturation, $\mathrm{PtcO}_{2}$ and $\mathrm{PtcCO}_{2}$ ). These surrogate endpoints were often altered but they could be easily normalized and did not require intervention by an anaesthesia nurse. The reason for not including the addition of oxygen was to improve the sensitivity to detect respiratory effects of the protocol used. As more than $50 \%$ of the patients required extra oxygen to reach normal oxygenation values during the procedure this assumption was verified. Further, it underlines potential risks with this type of technique if precautions are not made such as the ones suggested by the recently presented European Guidelines for sedation and analgesia by non anaesthesiology doctors and which were also incorporated when constructing the present protocol [11].

\subsection{Pulmonary Data}

Pulse oximetry is commonly used, although the accuracy is decreased with descending values. In order to improve reliability we therefore also used transcutaneous partial pressure measurement for oxygen and carbon dioxide, which are known to be accurate $[18,19]$. Pulse oximetry and transcutaneous measurement together made us able to better estimate the effect of PCS on patient oxygenation and ventilation. Eighteen of the 34 patients in this study showed signs of impaired ventilation as indicated by oxygen desaturation, tissue hypoxaemia, hypercarbia, or low respiratory rate. The use of oxygen during PCS is therefore desirable if level of saturation is $>90 \%$ because of the risk of hypoxia and oxygen deficit at the tissue level [21,22]. However, one must consider habitually lower oxygen saturation among elderly patients [19]. In neonates $\mathrm{PaO}_{2}$ is equal to $\mathrm{PtcO}_{2}$ but with the more keratinised epidermis in adults, there is a greater gradient. We focused on changes in values during the whole procedure and, of course, the extremes when more than one variable was affected. The low initial $\mathrm{PtcO}_{2}$ may in some cases be a result of technical problems in application of the sensor, which should preferably be placed near the heart, over the clavicle on thin skin. Dermal perfusion is another limitation for accurate recording of $\mathrm{PtcO}_{2}$. $\mathrm{PtcCO}_{2}$ is less affected due to the high solubility of carbon dioxide. However, we found hypercarbia in two patients who also had decreased $\mathrm{PtcO}_{2}$. This indicates that PCS is a potent technique for sedation with obvious effect on respiratory function, and the inclusion and exclusion criteria of patients for PCS given in the Guidelines for sedation... [11] are recommended.

In this study we combined two drugs. Due to interactivity a combination of drugs increase the risk of cardiopulmonary instability. The combination of remifentanil and propofol when used for PCS for ESWL has been shown to increase the risk of oxygen desaturation and apnoeic episodes, compared with propofol alone [23]. The same has been shown for alfentanil and propofol, where $40 \%$ of the patients [24] developed respiratory depression after alfentanil $15 \mu \mathrm{g} / \mathrm{kg}$. The induction dose of alfentanil in our study was $1.7 \mu \mathrm{g} / \mathrm{kg}$, and $10 \mu \mathrm{g} / \mathrm{kg}$ for the whole procedure.

It is debatable to put PCS in non-anaesthesiology trained hands using a combination of alfentanil and propofol as alfentanil is known to have a strong respiratory depressive effect which shows a considerable and unpredictable person-to-person variability. Alfentanil also decreases respiratory rate [25]. These effects are known to be augmented by the decrease in tidal volume caused by propofol [26]. Despite these potential deleterious effects, the normalization of the respiratory variables was easily accomplished without the intervention by the anaesthetic nurse. This supports the use of the technique as suggested when adhering to guidelines for sedation. [11].

\subsection{Cardiovascular Data}

The combination of propofol and alfentanil had little and limited effect on cardiovascular variables. The safety limits for blood pressure (MAP $>70)$ and heart rate (HR $>50$ ) were breached in only one patient, presumably because of the small doses of propofol and alfentanil given, combined with the rapid redistribution. The cardiac depressing effect and vasodilatation of propofol becomes more prominent with increasing doses. The mean induction dose was one-tenth of the usual dose for induction of anaesthesia, and the mean total dose of propofol 
for ESWL was $0.9 \mathrm{mg} / \mathrm{kg}$. However, MAP $>70$ is considered to be a reasonable target for maintaining adequate organ perfusion in most patients [27,28]. Except for in one patient our PCS setting fulfilled this, but our data indicated that 18 patients had signs of descending concentrations of oxygen in tissue, despite a MAP of $>70$.

\subsection{Sedation}

Doses of propofol and alfentanil were rather small in total, but enough to sedate for comfort. Some patients reached a deep state of sedation with BIS values of around 70 . These levels would be expected from a system that patients find satisfying, and have been shown in a previous study of PCS in burns [8]. Monitoring of sedation also involved a sedation scale, on the basis of interaction with the patient, which is of more clinical use and may contribute to greater comfort. An interactive sedation scale also stimulates the patients, which affect safety of the sedation. Although some of our patients were heavily sedated, they were always able to respond to verbal stimuli. The patients did not always talk, but were able to respond saying yes or no etc. For safety reasons we consider verbal contact being of great importance and absolutely essential, when PCS is handled by non-anaesthesiology doctors.

\subsection{PCS System}

We gave a small induction dose of propofol and alfentanil because the onset of pain and discomfort is quick when ESWL is started. During the procedure the level of pain is more or less constant, but the patients were able to give themselves additional doses when necessary. Three patients made demands that widely exceeded the doses delivered (ratio > 3.8). High intensity of pain made these patients push the delivery button two or three times during the dose delivery period, when doses can't be effectuated. Comparative studies on PCS where demand/ delivery ratio is presented are few, and the usage of different drugs and doses also bias the comparison. However, PCS during ESWL showed a ratio of 1.60 using propofol and remifentanil [23] and PCS using propofol for dental treatment resulted in a ratio of 1.51 [15], and patient-controlled analgesia with morphine for post operative pain control reports a ratio between 1.35 and 1.76 $[29,30]$. All these studies also evaluated PCS as preferred or satisfying by the patient. Ratios of up to 1.8 , and even more, may not influence on the overall satisfaction of PCS, as all patients in our study wished to have PCS if they required lithotripsy in future.

\subsection{Implementation in the Clinic}

So far, the ESWL-team has performed in total 2779 pro- cedures with the presented PCS-concept, with no serious events requiring consultation outside the ESVL-team. All patients have responded to verbal stimuli and decreasing levels of saturation is corrected with oxygen on nose catheter and/or verbal stimuli. All interventions in alignment with the guidelines for sedation... [11].

\section{Conclusions}

PCS can be used during ESWL with good patient satisfaction. From a safety perspective it is noteworthy that, when combining the anaesthetic propofol and alfentanil, anticipated effects on the respiration such as hypoxia or hypercarbia or in some cases both, were observed. With a minor intervention, by non-anaesthesiology personal, which included provision of oxygen, normalization of vital signs was accomplished.

Limitations: the strength of the conclusions in this study is hampered by the small sample size (for a safety evaluation) the observational study design without comparison of different regimens, and the transcutaneous gas analysis on adults that may display inaccurate values for oxygen and carbon dioxide. Despite these limitations we wish to display the need for adequate interventions with a propofol and alfentanil PCS-system, due to trends pointing at increased levels of carbon dioxide, respiratory depression and oxygen desaturation. Our results can be used for set up in a specific clinical situation.

\section{REFERENCES}

[1] J. A. Alhashemi and A. M. Kaki, "Anesthesiologist-Controlled versus Patient-Controlled Propofol Sedation for Shockwave Lithotripsy," Canadian Journal of Anesthesia, Vol. 53, No. 5, 2006, pp. 449-455. doi:10.1007/BF03022616

[2] M. A. Burmeister, et al., "A Comparison of Anaesthetic Techniques for Shock Wave Lithotripsy: The Use of a Remifentanil Infusion Alone Compared to Intermittent Fentanyl Boluses Combined with a Llow Dose Propofol Infusion," Anaesthesia, Vol. 59, No. 9, 2002, pp. 877 881. doi:10.1046/j.1365-2044.2002.02820.x

[3] M. Coloma, et al., "Fast-Tracking after Immersion Lithotripsy: General Anesthesia versus Monitored Anesthesia Care," Anesthesia \& Analgesia, Vol. 91, No. 1, 2000, pp. 92-96.

[4] T. G. Monk, J. M. Rater and P. F. White, "Comparison of Alfentanil and Ketamine Infusions in Combination with Midazolam for Outpatient Lithotripsy," Anesthesiology, Vol. 74, No. 6, 1991, pp. 1023-1028. doi:10.1097/00000542-199106000-00009

[5] E. Bright, et al., "Patient-Controlled Sedation for Colonoscopy: A Randomized Trial Comparing Patient-Controlled Administration of Propofol and Alfentanil with Physician-Administered Midazolam and Pethidine," Endoscopy, Vol. 35, No. 8, 2003, pp. 683-687. 
doi:10.1055/s-2003-41519

[6] I. A. Herrick, et al., "Patient-Controlled Propofol Sedation for Elderly Patients: Safety and Patient Attitude toWard Control," Canadian Journal of Anesthesia, Vol. 43, No. 10, 1996, pp. 1014-1018. doi:10.1007/BF03011902

[7] R. G. Dell and A. H. Cloote, "Patient-Controlled Sedation during Transvaginal Oocyte Retrieval: An Assessment of Patient Acceptance of Patient-Controlled Sedation Using a Mixture of Propofol and Alfentanil," European Journal of Anaesthesiology, Vol. 15, No. 2, 1998, pp. 210-215.

[8] C. Coimbra, M. Choiniere and T. M. Hemmerling, "Patient-Controlled Sedation Using Propofol for Dressing Changes in Burn Patients: A Dose-Finding Study," Anesthesia \& Analgesia, Vol. 97, No. 3, 2003, pp. 839-842. doi:10.1213/01.ANE.0000074236.76333.53

[9] C. A. Stonell, K. Leslie and A. R. Absalom, "Effect-Site Targeted Patient-Controlled Sedation with Propofol: Comparison with Anaesthetist Administration for Colonoscopy," Anaesthesia, Vol. 61, No. 3, 2006, pp. 240-247. doi:10.1111/j.1365-2044.2005.04509.x

[10] A. Nilsson, et al., "Patient Controlled Sedation Using a Standard Protocol for Dressing Changes in Burns: Patients' Preference, Procedural Details and a Preliminary Safety Evaluation," Burns, Vol. 34, No. 7, 2008, pp. 929934.

[11] J. T. A. Knape, et al., "Guidelines for Sedation and/or Analgesia by Non-Anaesthesiology Doctors: Section and Board of Anaesthesiology1. European Union of Medical Specialists," European Journal of Anaesthesiology, Vol. 24, No. 7, 2007, p p. 563-567. doi:10.1017/S0265021506002092

[12] C. Larsson and P. Staun, "Evaluation of a New FibreOptical Monitor for Respiratory Rate Monitoring," Journal of Clinical Monitoring and Computing, Vol. 15, No. 5, 1999, pp. 295-298. doi:10.1023/A:1009967122272

[13] P. S. Myles, et al., "Bispectral Index Monitoring to Prevent Awareness during Anaesthesia: The B-Aware Randomised Controlled Trial," The Lancet, Vol. 363, No. 9423 , 2004, pp. 1757-1763. doi:10.1016/S0140-6736(04)16300-9

[14] J. Liu, H. Singh and P. F. White, "Electroencephalographic Bispectral Index Correlates with Intraoperative Recall and Depth of Propofol-Induced Sedation," Anesthesia \& Analgesia, Vol. 84, No. 1, 1997, pp. 185-189.

[15] V. L. Oei-Lim, et al., "Patient-Controlled versus Anesthesiologist-Controlled Conscious Sedation with Propofol for Dental Treatment in Anxious Patients," Anesthesia \& Analgesia, Vol. 86, No. 5, 1998, pp. 967-972.

[16] E. N. Evans, K. Ganeshalingam and P. Ebden, "Changes in Oxygen Saturation and Transcutaneous Carbon Dioxide and Oxygen Levels in Patients Undergoing Fibreoptic Bronchoscopy," Respiratory Medicine, Vol. 92, No. 5, 1998, pp. 739-742. doi:10.1016/S0954-6111(98)90005-1

[17] J.-P. Janssens, et al., "Non-Invasive (Transcutaneous) Monitoring of $\mathrm{PCO}_{2}\left(\mathrm{TcPCO}_{2}\right)$ in Older Adults," Gerontology, Vol. 51, No. 3, 2005, pp. 174-178.

\section{doi: $10.1159 / 000083990$}

[18] B. W. Palmisano and J. W. Severinghaus, "Transcutaneous $\mathrm{PCO}_{2}$ and $\mathrm{PO}_{2}$ : A Multicenter Study of Accuracy," Journal of Clinical Monitoring and Computing, Vol. 6, No. 3, 1990, pp. 189-195. doi:10.1007/BF02832145

[19] K. K. Tremper and S. J. Barker, "Transcutaneous Oxygen Measurement: Experimental Studies and Adult Applications," International Anesthesiology Clinics, Vol. 25, No. 3, 1987, pp. 67-96. doi:10.1097/00004311-198702530-00006

[20] D. D. Külling, et al., "Safer Colonoscopy with PatientControlled Analgesia and Sedation with Propofol and Alfentanil," Gastrointestinal Endoscopy, Vol. 54, No. 1, 2001, pp. 1-7. doi:10.1067/mge.2001.116174

[21] I. L. Tyler, et al., "Continuous Monitoring of Arterial Oxygen Saturation with Pulse Oximetry during Transfer to the Recovery Room," Anesthesia \& Analgesia, Vol. 64, No. 11, 1985, pp. 1108-1112. doi:10.1213/00000539-198511000-00013

[22] K. K. Tremper and S. J. Barker, "Pulse Oximetry," Anesthesiology, Vol. 70, No. 1, 1989, pp. 98-108. doi:10.1097/00000542-198901000-00019

[23] H. S. Joo, et al., "A Comparison of Patient-Controlled Sedation Using Either Remifentanil or Remifentanil-Propofol for Shock Wave Lithotripsy," Anesthesia \& Analgesia, Vol. 93, No. 5, 2001, pp. 1227-1232. doi:10.1097/00000539-200111000-00037

[24] J. B. Yee, et al., "Propofol and Alfentanil for Sedation during Placement of Retrobulbar Block for Cataract Surgery," Journal of Clinical Anesthesia, Vol. 8, No. 8, 1996, pp. 623-626. doi:10.1016/S0952-8180(96)00137-7

[25] M. N. Avramov and P. F. White, "Use of Alfentanil and Propofol for Outpatient Monitored Anesthesia Care: Determining the Optimal Dosing Regimen," Anesthesia \& Analgesia, Vol. 85, No. 3, 1997, pp. 566-572.

[26] N. W. Goodman, A. M. S. Black and J. A. Carter, et al., "Some Ventilatory Effects of Propofol as Sole Anaesthetic Agent," British of Journal Anaesthesia, Vol. 59, No. 12, 1987, pp. 1497-1503. doi:10.1093/bja/59.12.1497

[27] L. M. Bigatello and E. George, "Hemodynamic Monitoring," Minerva Anestesiology, Vol. 68, No. 4, 2002, pp. 219-225.

[28] J. B. Henry, et al., "Mean Arterial Pressure (MAP): An Alternative and Preferable Measurement to Systolic Blood Pressure (SBP) in Patients for Hypotension Detection during Hemapheresis," Journal of Clinical Apheresis, Vol. 17, No. 2, 2002, pp. 55-64. doi:10.1002/jca.10022

[29] C. W. Cheung, et al., "An Audit of Postoperative Intravenous Patient-Controlled Analgesia with Morphine: Evolution over the Last Decade," European Journal of Pain, Vol. 13, No. 5, 2008, pp. 464-471.

[30] F. Kucukemre, et al., "Remifentanil Compared with Morphine for Postoperative Patient-Controlled Analgesia after Major Abdominal Surgery: A Randomized Controlled Trial," European Journal of Anaesthesiology, Vol. 22, No. 5, 2005, pp. 378-385. doi:10.1017/S0265021505000657 